\title{
Intramuscular Pressure Is Almost Three Times Higher in Fibromyalgia Patients: A Possible Mechanism for Understanding the Muscle Pain and Tenderness
}

\author{
Robert S. Katz ${ }^{1}$, Frank Leavitt ${ }^{2}$, Alexandra Katz Small ${ }^{3}$, and Ben J. Small ${ }^{4}$
}

\begin{abstract}
Objective. Widespread pain in fibromyalgia syndrome (FMS) is conventionally viewed as arising from disordered central processing. This study examines intramuscular pressure in the trapezius as an alternative mechanism for understanding FMS pain.

Methods. One hundred eight patients who satisfied the American College of Rheumatology criteria for FMS and 30 patients who met the ACR criteria for another rheumatic disease comprised the study groups. Muscle pressure was measured in $\mathrm{mmHg}$ using a pressure gauge attached to a no. 22 needle inserted into the mid-portion of the trapezius muscle. In addition, patients with FMS and rheumatic disease controls had dolorimetry testing, digital palpation, and reported pain scores.

Results. Muscle pressure was substantially higher in patients with FMS with a mean value of 33.48 $\pm 5.90 \mathrm{mmHg}$. Only 2 of 108 patients had muscle pressure of $<23 \mathrm{mmHg}$. The mean pressure in rheumatic disease controls was $12.23 \pm 3.75 \mathrm{mmHg}$, with a range from 3-22 mmHg. Patients with FMS were more tender than controls based on both dolorimetry $(P<0.001)$ and digital palpation $(P<0.001)$. The mean pain score in patients with FMS and controls was $6.68 \pm 1.91$ and $1.43 \pm 1.79$, respectively $(P<0.001)$.

Conclusion. Pressure in the trapezius muscle of patients with FMS is remarkably elevated and may be an intrinsic feature of FMS that could be monitored as part of the diagnostic evaluation. The burden of the pressure abnormality may help explain the diffuse muscle pain of FMS. Therefore, FMS as a disorder of exclusively central pain processing should be revisited. Therapeutically, the reduction of muscle pressure may change the clinical picture significantly.
\end{abstract}

Key Indexing Terms: central sensitization, fibromyalgia, intramuscular pressure, pain, trapezius muscle

Central sensitization theory, the prevailing account of chronic pain in fibromyalgia syndrome (FMS $)^{1}$, makes a fundamental distinction between the site of pain and the source of pain. According to this theory, they are not the same ${ }^{2}$. Abnormal processing of sensations by neural brain activity is the presumed mechanism for turning on pain at body sites that are at a distance from the source. This formulation guides treatment and influences the development of new medication. Currently, the first-line medications for pain in FMS are centrally acting, including pregabalin, duloxetine, and milnaciprin. They either decrease glutamate or increase serotonin and norepinephrine to try to ease the presumed central nervous system effect on widespread musculoskeletal pain ${ }^{3,4}$.

${ }^{1}$ R.S. Katz, MD, Section of Rheumatology, Department of Internal Medicine, Rush Medical College; ${ }^{2}$. Leavitt, PhD, Department of Behavioral Sciences, Rush Medical College; ${ }^{3}$ A.K. Small, MD, Rush Medical College; ${ }^{4}$ B.J. Small, $M D$, Northwestern University's Feinberg School of Medicine, Chicago, Illinois, USA.

The authors declare no conflicts of interest.

Address correspondence to Dr. R.S. Katz, 1725 W. Harrison St, Suite 365, Chicago, IL 60612, USA.Email: rkatzchil@gmail.com.

Full Release Article. For details see Reprints and Permissions at jrheum.org. Accepted for publication September 5, 2020.
Our present study advances a new view of FMS pain that places the pain generator and the site of pain in the same peripheral location - the painful tender muscles. Specifically, this study challenges the prevailing theory of pain in FMS and seeks to show that pain in the muscles is linked to elevated pressure in the muscles.

At this point, there is no information on the normalcy of intramuscular pressure (IMP) in FMS. The purpose of this study was to evaluate the level of muscle tenderness and IMP in the trapezius muscle of patients with FMS compared to controls. In some studies, over $90 \%$ of the FMS sample report pain in the trapezius ${ }^{5}$; therefore, the trapezius muscle was chosen for study because it is a highly symptomatic site in patients with FMS that is easy to access and is likely to be representative of the pain they experience in other body sites.

\section{MATERIALS AND METHODS}

Participants in the study were recruited from a pool of consecutive patients in a high-volume rheumatologic clinical practice at Rush University Medical Center. This study was approved by the Rush University institutional review board (ORA 17052205). The goals and procedures of the study were fully explained and informed consent was signed prior to initiation of any study activities.

All potential participants were screened for eligibility criteria. At screening, demographic data were collected on potential participants. Patients who were proposed for the FMS group also completed the 
Fibromyalgia Survey Questionnaire to determine if they satisfied the modified 2010 and 2016 American College of Rheumatology (ACR) criteria for FMS for inclusion or exclusion ${ }^{6,7}$.

The 108 patients in the FMS group, aged 18-70 years and English speaking, were required to have an established clinical diagnosis of FMS confirmed by an experienced rheumatologist (RK) who performed a comprehensive clinical examination; patients must also have satisfied the ACR criteria for FMS diagnosis. The rheumatologist was unaware of the results of the tenderness examination or the patient's score on the ACR criteria. Patients were required to have pain in the trapezius for inclusion.

A control group was recruited from a pool of patients without evidence of FMS, aged 18-70 years and English speaking, and who fulfilled the criteria for other rheumatic diseases [including rheumatoid arthritis (RA), systemic lupus erythematosus (SLE), psoriatic arthritis (PsA), and Sjögren syndrome (SS)], which were confirmed by a rheumatologist. All patients agreed to undergo IMP testing. Patients who scored above the cutoff for the ACR criteria for FMS were excluded. Inclusion criteria for the control group were met by 30 patients.

The physical measurements followed a standard protocol. Measurements were carried out by an examiner experienced in testing for muscle tenderness and pressure; these were conducted while standing behind the subject. Recordings were always taken at rest, with the subject in a seated position, and the head in a neutral position staring straight ahead. The examination site was always the midportion of the trapezius muscle.

Pain. Pain intensity in the trapezius muscle was measured on a $0-10$ visual analog scale (VAS) of pain intensity, with anchor points of 0 (no pain) and 10 (the most unbearable pain imaginable). The VAS is commonly used to assess pain with high reliability ${ }^{8}$.

Muscle tenderness. The level of muscle tenderness was graded according to
2 conventionally used measures: digital palpation and dolorimetry. Digital palpation of the trapezius muscle was carried out with subjects in the seated position. Digital pressure was steadily applied with 2 fingertips for a period of 4 seconds by a nurse highly trained in digital palpation who was not aware of patient diagnosis. The level of discomfort was graded on a validated 4-point scale: 0, no tenderness (no discomfort or pain reported at the site of palpation); 1, mild tenderness (pain reported at the site of palpation, no observable reaction otherwise); 2 , moderate tenderness (pain with a grimace and/or flinch on palpation); and 3, severe tenderness (pain with marked withdrawal on palpation)?.

Muscle tenderness was also assessed with a 30-lb FDK forced gauge dolorimeter (Wagner Instruments) calibrated in 0.25-lb increments with the disc head vertically positioned over the trapezius muscle. Pressure was applied at a slow rate with patients instructed to indicate when they experienced pain. The point at which applied pressure transitioned to pain, also known as the pressure/pain threshold (PPT), was recorded in pounds.

$I M P$. IMP was measured with a Stryker pressure gauge (used to diagnose anterior compartment syndrome) attached to a no. 22 needle. Saline $(0.3 \mathrm{cc})$ was injected into the midportion of the trapezius muscle at an angle of $70^{\circ}$, to a depth of $2 \mathrm{~cm}$ from the skin surface. Muscle pressure was recorded in $\mathrm{mmHg}$ (Figure 1).

Statistical analysis. The primary variables for analysis were age, sex, pain in the trapezius muscle, tenderness based on digital palpation and dolorimetry, and IMP. Data are presented as mean $( \pm S D)$. Differences between groups on continuous variables were analyzed by $t$-tests. Differences among more than 2 groups were analyzed by 1-way ANOVA. Associations between variables on categorical data were analyzed by the chi-square test. $P$ values $<0.05$ were considered significant. Sample size was not calculated for this study, because the analyses are intended for hypothesis generation rather

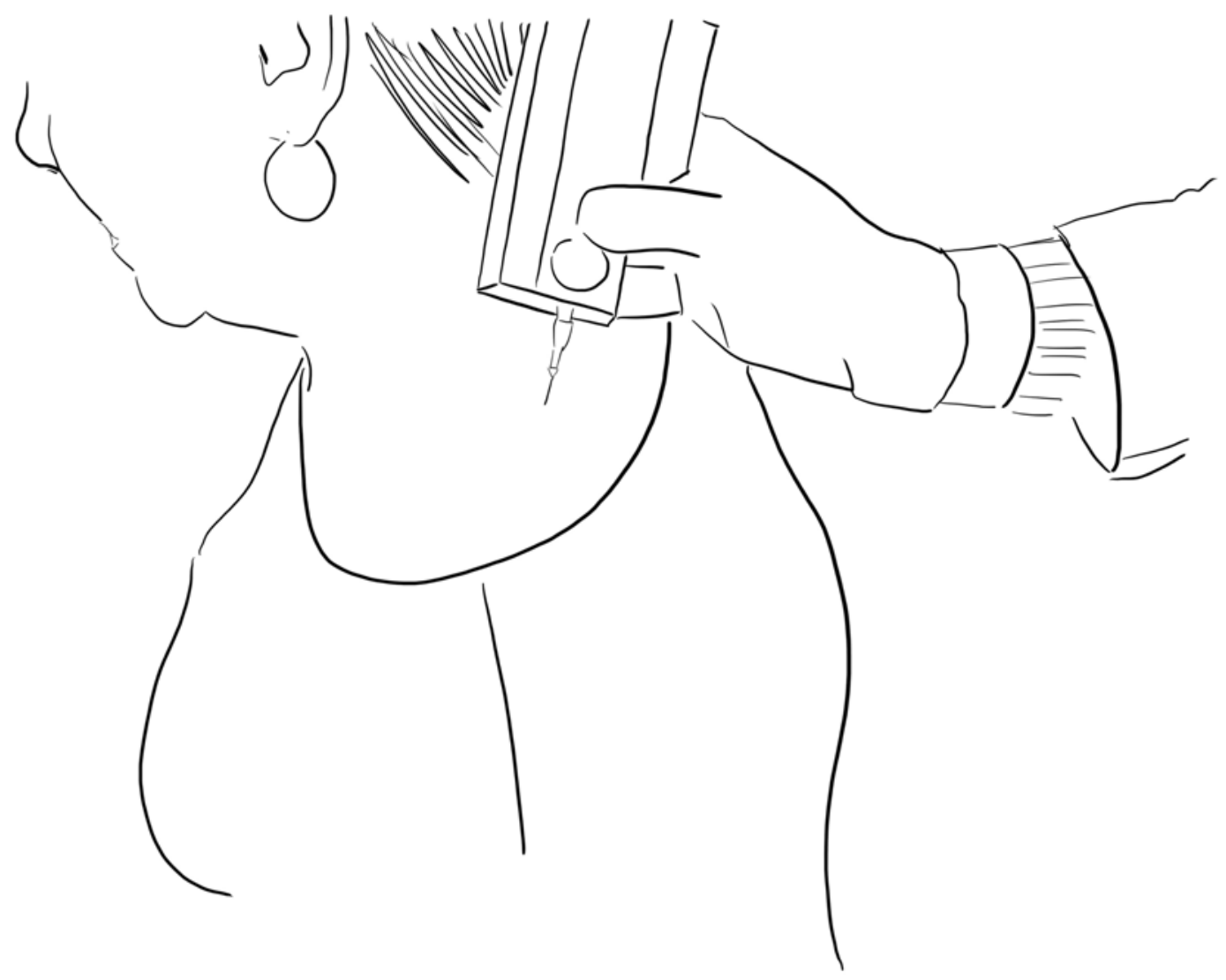

Figure 1. Line drawing demonstrating muscle pressure measurement technique. 
than confirmation. All $P$ values and summary statistics are intended to be descriptive.

\section{RESULTS}

Patients in the FMS and control groups were well matched for age, with mean ages of $48.3 \pm 13.9$ and $47.9 \pm 10.3$, respectively $(P=0.87)$. In total, $84 \%(91 / 108)$ of the patients with FMS group were female. This is significantly higher than the $66.7 \%$ $(20 / 30)$ in the control sample $(P<0.05)$. Additionally, the control group contained $50 \%(15 / 30)$ patients who satisfied the ACR criteria for RA, 23\% (7/30) who satisfied criteria for SLE, $17 \%$ (5/30) who satisfied criteria for PsA, and 10\% (3/30) who satisfied criteria for SS (data not shown)

Pain was moderately high in the FMS group, with a mean score of $6.68 \pm 1.91$. Pain in the control group was substantially less, with a mean score of $1.43 \pm 1.79$. The significantly higher pain score in the FMS group $(P<0.001)$ was expected, as pain was an inclusion criterion for the FMS group (Table 1).

Tenderness was significantly higher in the FMS group whether measured by dolorimetry or digital palpation. The mean PPT score (measured by dolorimetry) was 14.5 lbs less in the FMS group compared to the control group (8.03 vs 22.54, $P<0.001)$. The lower the value at which pressure shifts to pain, the greater the tenderness. On digital palpation, patients with FMS had an average score of $2.09 \pm 0.73$, while controls had an average score of $0.47 \pm 0.78(P<0.001)$. High scores on digital palpation indicate significant tenderness.
Pressure in the trapezius muscle was extremely elevated in patients with FMS, with an average pressure of 33.48 $\pm 5.90 \mathrm{mmHg}$. Trapezius muscle pressure in controls was significantly lower, with an average pressure of $12.23 \pm 3.75 \mathrm{mmHg}$ $(P<0.001)$. IMP in the FMS group ranged from 8 to $58 \mathrm{mmHg}$, with only 2 values less than $23 \mathrm{mmHg}$. IMP in controls ranged from 3 to $22 \mathrm{mmHg}$ (Table 1 ). The higher the pain score, the greater the pressure in the trapezius muscle (Figure 2).

There was no morbidity associated with the determination of muscle pressure with a pressure gauge and needle. Although pneumothorax could be a complication of the procedure, it was not seen in our study. Some patients had a temporary increase in muscle soreness from the procedure, which includes injecting a small amount of saline into the trapezius muscle. Muscle pressure determination was similar to the tender point injections we occasionally give our FMS patients. Patients were able to tolerate the procedure with little or no complaint.

An assumption can be made that measuring muscle pressure in areas throughout the body, including the lower extremities, would also demonstrate greater muscle pressure than in controls, but the trapezius muscle was chosen because of convenience.

Sex did not affect results because there were no significant differences in muscle pressure for males and females. The average muscle pressure of males and females was $34.4 \pm 4.8$ and 33.3 \pm 6.1 , respectively $(P=0.48)$.

Muscle pressure in the trapezius did not vary significantly with age for the study population. For those in the 3 age categories

Table 1. Clinical features of 108 patients with FM and 30 non-FM controls.

\begin{tabular}{lcccc}
\hline & FM & Controls & $95 \% \mathrm{CI}$ & $P$ \\
\hline Pain $^{\mathrm{a}}$ & $6.68 \pm 1.91$ & $1.43 \pm 1.79$ & $4.47-6.01$ & $<0.001$ \\
Tenderness & & & & \\
$\quad$ Digital palpation & $2.09 \pm 0.73$ & $0.47 \pm 0.78$ & $1.32-1.93$ & $<0.001$ \\
$\quad$ Dolorimeter & $8.03 \pm 4.06$ & $22.54 \pm 8.45$ & -16.67 to -12.35 & $<0.001$ \\
Intramuscular pressure & $33.48 \pm 5.90$ & $12.23 \pm 3.75$ & $19.0-23.5$ & $<0.001$ \\
\hline
\end{tabular}

Values are mean \pm SD. ${ }^{a}$ Pain in trapezius was an inclusion criterion for the FM sample. FM: fibromyalgia.

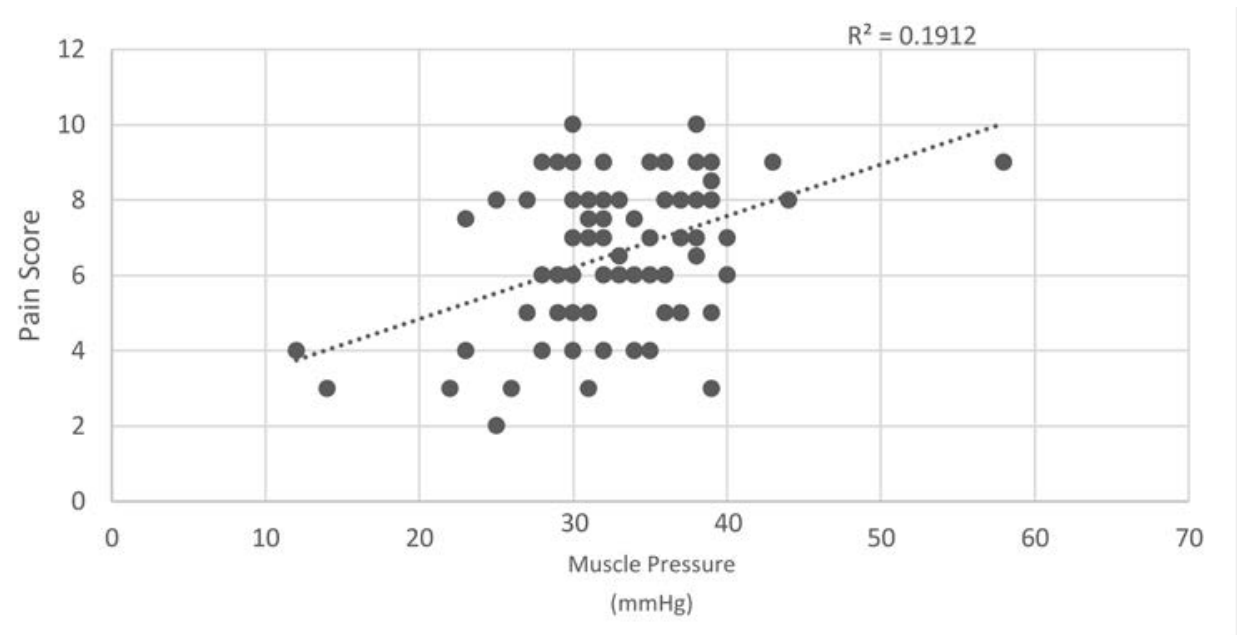

Figure 2. FMS pain score vs muscle pressure (all FMS patients). FMS: fibromyalgia syndrome. 
$(<40,40-59,>59 \mathrm{yrs})$, the average pressure values were 34.0 $\pm 5.7,33.3 \pm 6.6,33.1 \pm 4.2 \mathrm{mmHg}$, respectively $(P=0.70$; data not shown).

There was excellent correlation between muscle pressure, pain level, dolorimetry, and manual muscle tenderness (Table 2).

\section{DISCUSSION}

In the most striking finding, the magnitude of pressure in the trapezius of patients with FMS at rest was almost $22 \mathrm{mmHg}$ greater than the pressure of controls. In $98 \%$ of FMS cases, a pressure reading of $\geq 23 \mathrm{mmHg}$ was recorded. A resting value in the area of $8 \mathrm{mmHg}$ is considered normal based on an older study that used a different technique ${ }^{10}$. Surprisingly, patients with FMS may be functioning with a magnitude of pressure that is significantly elevated compared to controls, yet this burden is not evident in the large volume of studies addressing muscle pathology in FMS ${ }^{11}$.

The distribution of values in the 2 groups had almost no overlap. IMP in the FMS group ranged from 8 to $58 \mathrm{mmHg}$, with only 2 values $<23 \mathrm{mmHg}$. Pressure in the controls ranged from 3 to $22 \mathrm{mmHg}$. The differences are sufficiently large to consider muscle pressure as a potential diagnostic discriminator. In this study, elevated IMP was specific to FMS among the rheumatologic population. If hyperpressure in the trapezius can be confirmed by other investigators, then quantifying IMP may be of value as a potential biomarker for FMS.

Abnormal pain tenderness was also observed in association with increased pressure in the trapezius muscle of FMS patients. The PPT score (measured by dolorimetry) for patients with FMS is much lower than in controls when using both digital and dolorimeter testing. The findings are in line with previous findings showing pain and tenderness to be common in the trapezius muscle in $\mathrm{FMS}^{12}$. Pain in the FMS sample was also elevated, but the study design biased this result. However, pain and muscle tenderness have been observed in association with hyperpressure in other body sites, suggesting a critical linkage ${ }^{10,13}$. Understanding the overlapping nature of these variables may be key in unlocking a greater understanding of muscle pain in FMS.

The trapezius is a large muscle that runs from the upper back to the neck and shoulders ${ }^{14}$ and covers areas of the body that are considered primary pain sites in $\mathrm{FMS}^{15}$. Considering the compressing effects of extended pressure on the small capillaries in muscles, elevated pressure in the trapezius could be a mechanism for diffuse muscle pain. Abnormally high IMP is known to compress capillaries, which in turn obstructs blood flow and thereby prevents adequate oxygenation of muscle tissue, a condition well known to set off and maintain pain sensations ${ }^{10,16}$. Muscle biopsy studies have shown evidence of hypoxia and no inflammation ${ }^{11,16,17}$. It is possible that increased muscle pressure could be compressing capillaries and reducing blood flow to muscle. The burden of hyperpressure in the trapezius over time is likely to affect health more broadly and could be the mechanism for the muscle pain and fatigue that characterize FMS ${ }^{18,19,20}$.

Contrary to current beliefs that reject muscle abnormality in $\mathrm{FMS}^{11}$, the conspicuous elevation in IMP at rest raises the possibility that pain and other symptoms of FMS arise from ongoing pressure anomalies in the trapezius muscles of patients with FMS, and not at the level of central sensitization. In our view, myalgias are associated with elevated muscle pressure rather than damage or central sensitization. Based on the current data, the central sensitization theory may be leading investigators to focus on only 1 treatment target. Finding muscle pressure 3 times greater versus controls suggests the importance of targeting

Table 2. Correlation between muscle pressure, pain level, dolorimetry, and muscle tenderness.

\begin{tabular}{|c|c|c|c|c|c|c|}
\hline Correlation coefficient & 0.63 & 0.61 & -0.70 & 0.73 & -0.62 & -0.58 \\
\hline Fisher-transformed correlation & 0.74 & 0.70 & -0.86 & 0.93 & -0.72 & -0.66 \\
\hline Total pairs, n & 130 & 130 & 130 & 130 & 130 & 130 \\
\hline s & 0.07 & 0.07 & 0.06 & 0.06 & 0.07 & 0.07 \\
\hline Fisher-transformed $s\left(s^{\prime}\right)$ & 0.09 & 0.09 & 0.09 & 0.09 & 0.09 & 0.09 \\
\hline $\mathrm{t}$ & 9.30 & 8.70 & -11.11 & 12.13 & -8.92 & -8.05 \\
\hline Fisher-transformed lower 95\% CI & 0.57 & 0.53 & -1.04 & 0.75 & -0.89 & -0.83 \\
\hline Fisher-transformed upper 95\% CI & 0.92 & 0.88 & -0.69 & 1.10 & -0.54 & -0.48 \\
\hline Correlation lower 95\% CI & 0.52 & 0.48 & -0.78 & 0.64 & -0.71 & -0.68 \\
\hline
\end{tabular}

Spearman correlations are used when at least 1 of the variables is ordinal. Pearson correlations are used when both variables are continuous (in the case of dolorimetry and muscle tension). A correlation coefficient of $\sim 0.6$ is considered a moderate positive relationship between 2 variables. A correlation coefficient of $\sim 0.8$ is considered a fairly strong positive relationship. The highest correlation is between pain and tenderness, which is intuitive. The highest of the muscle tension vs XX correlations was between muscle tensions and dolorimetry ( $\sim .7$, a moderately strong negative relationship). The correlation is negative, which indicates that as muscle tension increases, dolorimetry score decreases. The CI around all the correlation coefficients do not include zero and are therefore considered statistically significant correlations. Df: degrees of freedom; z-crit: critical value for z. 
muscle tension/pressure in addition to central (brain) mechanisms. This study directs attention to a peripheral target for both diagnosis and treatment that is not routinely monitored in clinical practice: intramuscular pressure. Hopefully, our current study also provides a revised roadmap for a better understanding of pain in FMS.

However, the finding of increased IMP does not disprove the major current pathophysiological hypothesis of central sensitization. There certainly may be both peripheral and central mechanisms causing the pain of FMS. In any case, increased muscle pressure might represent a diagnostic aid in FMS, as well as a target for treatment to reduce muscle pressure.

Although muscle pressure measurement is an important procedure in the diagnosis and possible etiology of FMS, dolorimetry might be substituted as an easier, less invasive quantitative measure once we determine that muscle pressure is elevated using the pressure gauge.

The study had several limitations. It was conducted on patients in treatment at a large tertiary medical center. The results may have been different if it were conducted with a wider spectrum of patients with FMS. Second, measurement of IMP was carried out at a single site, whereas pain presents across the entire trapezius muscle. Pressure at different sites may vary. Third, results regarding pain intensity differences between groups are notably exaggerated due to requiring FMS patients to have existing pain in their trapezius muscle for inclusion in the study. Finally, the control sample was fairly small relative to the FMS sample; however, the difference between groups was highly substantial and unlikely to change by adding additional subjects to the control group.

To our knowledge, this is the first study to show evidence of an abnormality in muscle pressure in FMS. The appreciation of elevated trapezius muscle pressure as a mechanism for hypoperfusion and diffuse muscle pain in FMS is important and suggests a target for both evaluation and intervention. Hyperpressure in the trapezius could become a useful test in evaluating FMS. In addition, therapeutic attempts to reduce muscle pressure, using a muscle pressure gauge as a guide, might help to evaluate more effective therapies for this widespread pain syndrome. Methods to reduce IMP may be therapeutic.

\section{REFERENCES}

1. Meeus M, Nijs J. Central sensitization: a biopsychosocial explanation for chronic widespread pain in patients with fibromyalgia and chronic fatigue syndrome. J Clin Rheumatol 2007:26;465-73.

2. Woolf CJ. Central sensitization: implications for the diagnosis and treatment of pain. Pain 2011;3 Suppl:S2-15.
3. Brown JP, Boulay LJ. Clinical experience with duloxetine in the management of chronic musculoskeletal pain: a focus on osteoarthritis of the knee. Ther Adv Musculoskelet Dis 2013; 5:291-304.

4. Lee JC, Nassikas NJ, Clauw DJ. The role of the central nervous system in the generation and maintenance of chronic pain in rheumatoid arthritis, osteoarthritis and fibromyalgia. Arthritis Res Ther 2011;13:211-6.

5. Friend R, Bennett RM. Distinguishing fibromyalgia from rheumatoid arthritis and systemic lupus in clinical questionnaires: an analysis of the revised fibromyalgia impact questionnaire (FIQR) and its variant the symptom impact questionnaire (SIQR) along with pain locations. Arthritis Res Ther 2011;13:R58.

6. Wolfe F, Clauw DJ, Fitzcharles MA, Goldenberg DL, Hauser W, Katz RS, et al. 2016 revisions to the 2010/2011 fibromyalgia criteria. Semin Arthritis Rheum 2016;46:319-29.

7. Wolfe F, Clauw DJ, Fitzcharles MA, Goldenberg DL, Hauser W, Katz RS, et al. Fibromyalgia criteria and severity scales for clinical and epidemiological studies: a modification of the ACR preliminary diagnostic criteria for fibromyalgia. J Rheumatol 2011;38:1113-22.

8. Bijur JP, Silver W, Gallagher EJ. Reliability of the visual analog scale for measurement of acute pain. Acad Emerg Med 2001;8:1153-7.

9. Hubbard DR, Berkoff GM. Myofascial trigger points show spontaneous needle EMG. Spine 1993;18:1803-7.

10. Hagert CG, Christenson JT. Hyperpressure in the trapezius muscle associated with fibrosis. Acta Orthop Scand 1990;61:263-5.

11. Simms RW. Is there muscle pathology in fibromyalgia syndrome? Rheum Dis Clin North Am 1996;22:245-66.

12. Maquet D, Croisier JL, Demoulin C, Crielaard JM. Pressure pain thresholds of tender point sites in patients with fibromyalgia and in healthy controls. Eur J Pain 2004;8:111-7.

13. Qvarfordt P, Christenson JT, Eklöf B, Ohlin P, Saltin B. Intramuscular pressure, muscle blood flow, and skeletal muscle metabolism in chronic anterior tibial compartment syndrome. Clin Orthop Relat Res 1983;179:284-90.

14. Johnson G, Bogduk N, Nowitzke A, House D. Anatomy and actions of the trapezius muscle. Clin Biomech 1994;9:44-50.

15. Henriksson KG. Muscle pain in neuromuscular disorders and primary fibromyalgia. Neurologija 1989;38:213-21.

16. Katz DL, Greene L, Ali A, Faridi Z. The pain of fibromyalgia syndrome is due to muscle hypoperfusion induced by regional vasomotor dysregulation. Med Hypotheses 2007;69:517-25.

17. Ruggiero L, Manganelli F, Santoro L. Muscle pain syndromes and fibromyalgia: the role of muscle biopsy. Curr Opin Support Palliat Care 2018;12:382-7.

18. Simms RW, Goldenberg DL, Felson DT, Mason JH. Tenderness in 75 anatomic sites. Distinguishing fibromyalgia from controls. Arthritis Rheum 1988;31:182-7.

19. Zidar J, Backman E, Bengtsson A, Henriksson KG. Quantitative EMG and muscle tension in painful muscles in fibromyalgia. Pain 1990;40:249-54.

20. Bengtsson A. The muscle in fibromyalgia. Rheumatology 2002;41:721-4. 


\section{Correction}

Intramuscular Pressure Is Almost Three Times Higher in Fibromyalgia Patients: A Possible Mechanism for Understanding the Muscle Pain and Tenderness

Robert S. Katz, Frank Leavitt, Alexandra Katz Small, and Ben J. Small. J Rheumatol 2021; doi:10.3899/jrheum.191068

In the Methods section, the injection of saline into the midportion of the trapezius muscle should be at an angle of $45^{\circ}$. The error does not affect the results or conclusions of the study.

doi:10.3899/jrheum.191068.C1 